\title{
Aiming for evidence-based best practices in thoracic surgery-leadership in surgery
}

Lung cancer remains a compelling cancer problem worldwide-according to data from the International Agency for Research on Cancer, over 2 million new cases of lung cancer will be diagnosed worldwide in 2018. More sobering are the 1.7 million deaths anticipated this year as a result of lung cancer. Many initiatives are underway to try and address this compelling public health crisis, including tobacco control initiatives, screening programmes and the development of better treatments. The particular details are beyond the scope of these introductory remarks. Surgery remains a key treatment for early stage cancers.

The legendary business guru Peter Drucker said that "Management is doing things right. Leadership is doing the right things." Thoracic surgeons, by virtue of their training are expert managers of lung cancer. As our understanding of surgery, recovery and the minimizing of treatment side effects has matured, we have embraced as a specialty the ideas and ideals of enhanced recovery after thoracic surgery (ERAS). We must lead the way in critically appraising elements of perioperative care to accurately identify the right things that need to be done for our patients.

Our contributors have outlined and ably discussed many areas that are emerging areas of perioperative leadership. Drs. Spicer and Fernando with their colleagues present their experience with ERAS protocols and outline the elements for successful implementation of ERAS protocol for thoracic surgical patients. Drs. McGuire, Yeung, French and colleagues tackle the vexing problem of prolonged air leak after pulmonary resection and provide some keen and innovative perspectives on best practices to consider. Drs. Gagne, Thompson and Villeneuve, together with their colleagues examine and discuss the elements of best perioperative patient care. Finally, Dr. Zielinski's group outlines the practical aspects of novel access techniques for oncologic lung resection.

We are grateful to our colleagues for their valuable contributions to this special edition and would like to recognize the editorial support of the board at $7 T D$. We feel that this collection of articles highlights the role that thoracic surgeons and their care partners in anaesthesia have as leaders in this domain; we hope that it serves as a catalyst and a guide to continue transforming and optimizing perioperative care for patients undergoing thoracic surgery.

\section{Acknowledgements}

None.

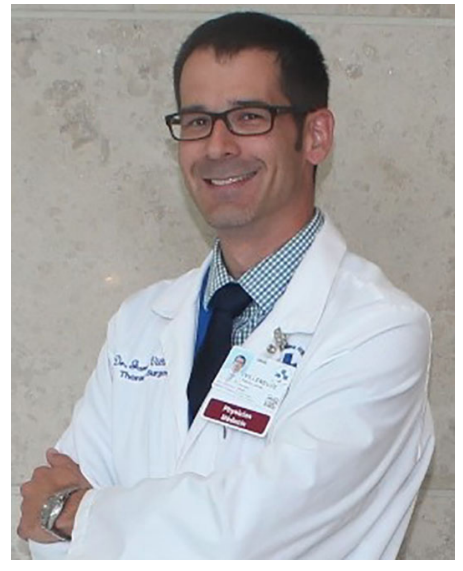

Patrick James Villeneuve

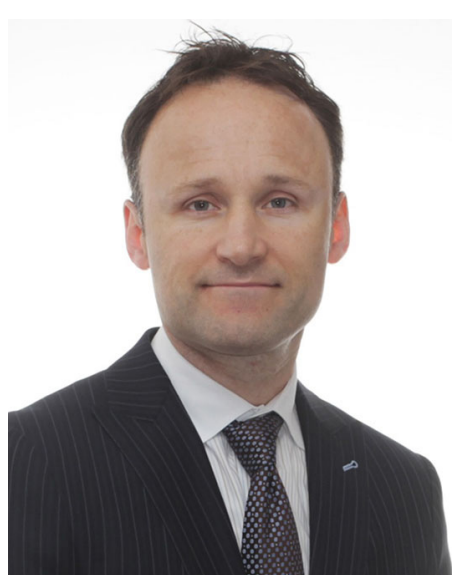

Sebastien Gilbert 


\section{Patrick James Villeneuve ${ }^{1,2}$ Sebastien Gilbert ${ }^{1,3}$ \\ ${ }^{1}$ Division of Thoracic Surgery, Department of Surgery, University of Ottawa, Ottawa, Ontario, Canada; ${ }^{2}$ Cancer Therapeutics Program, ${ }^{3}$ Clinical Epidemiology Program, Ottawa Hospital Research Institute, Ottawa, Ontario, Canada. \\ (Email: pvilleneuve@toh.ca; sgilbert@toh.ca) doi: $10.21037 /$ jtd.2018.10.121}

Conflicts of Interest: The authors have no conflicts of interest to declare. View this article at: http://dx.doi.org/10.21037/jtd.2018.10.121

Cite this article as: Villeneuve PJ, Gilbert S. Aiming for evidence-based best practices in thoracic surgery-leadership in surgery. J Thorac Dis 2018;10(Suppl 32):S3726-S3727. doi: $10.21037 /$ jtd.2018.10.121 